\title{
Commentaire sportif en direct : Etude des correspondances entre le rythme du jeu et le rythme de parole
}

\author{
Catherine Mathon, Gilles Boyé et Anna Kupść \\ CLLE-ERSSàB (UMR 5263) et Université Bordeaux Montaigne, France \\ \{catherine.mathon et gilles.boye\}@u-bordeaux-montaigne.fr
}

Résumé. L'étude que nous présentons ici s'inscrit dans la lignée des études portant sur les genres de discours oraux, et notamment sur les analyses prosodiques qui ont pu être proposées afin de définir les particularités phonostylistiques de plusieurs genres de discours et les discriminer les uns par rapport aux autres. Nous proposons donc une étude sur le rythme de parole dans un genre de discours particulier, celui du commentaire sportif en direct. Ce genre de discours nous intéresse en ceci que les productions linguistiques et phonostylistiques en sont particulièrement contraintes. L'une de ces contraintes concerne le contenu du commentaire : celui-ci est dicté directement par la nature de l'événement commenté. En effet, une partie du commentaire doit faire référence à l'action commentée. L'objectif de notre étude est de spécifier cette contrainte et de montrer comment celle-ci influe de manière générale sur différents aspects de l'organisation du discours (répartition de la parole en fonction du jeu et type de commentaires), aussi bien que sur les productions phonostylistiques des deux locuteurs principaux qui interviennent dans le commentaire, le journaliste et l'expert. Au niveau prosodique, nous nous intéressons principalement ici au rythme de parole des locuteurs, débit de parole et débit d'élocution, de manière à montrer l'influence potentielle du rythme du jeu sur le rythme du commentaire.

\begin{abstract}
This paper is inspired by the studies which concern oral discourse genres (phonogenres), especially in the domain of prosody, and which aim at providing descriptions of the phonostylistic properties for various phonogenres and to discriminate them based on prosodic features. Thereby we propose a study of speech rhythm in a specific phonogenre, namely TV live sport's report. We chose this specific phonogenre because of constraints which guide linguistic and phonostylistic productions. One of these constraints comes from the report's content: indeed the nature of the media event which is the object of the report (sport in our case) dictates the report's content (the journalist is going to speak about sport mostly, and more specifically about the sport actions which are shown at this particular moment). The aim of our study is to focus on this constraint and show how it affects different aspects of discourse organization: speech sharing between the journalist and the expert, the two main commentators, depending on game periods, and type of commentaries involved (play-by-play or color-commentary). We also show how discourse content influences phonostylistic productions of both speakers. In terms of prosody, we are interested principally in speech rhythm, i.e. speech rate and elocution rate. Our goal is to show the impact of sport rhythm on the rhythm of discourse (sport commentary).
\end{abstract}




\section{Introduction}

L'étude que nous présentons ici s'inscrit dans la lignée des études portant sur les genres de discours oraux, et notamment sur les analyses prosodiques qui ont pu être proposées afin de définir les particularités phonostylistiques de plusieurs genres de discours et les discriminer les uns par rapport aux autres. Nous nous sommes concentrés sur un genre de discours particulier, celui du commentaire sportif en direct. Ce genre de discours nous intéresse en ceci que les productions linguistiques et phonostylistiques en sont particulièrement contraintes. L'objectif de notre étude est de définir ces contraintes et de montrer comment celles-ci influent de manière générale sur différents aspects de l'organisation du discours (répartition de la parole en fonction du jeu et type de commentaires), aussi bien que sur les productions phonostylistiques des deux locuteurs principaux qui interviennent dans le commentaire, le journaliste et l'expert. Au niveau prosodique, nous nous intéressons principalement ici au rythme de parole des locuteurs, débit de parole et débit d'élocution, de manière à montrer l'influence potentielle du rythme du jeu sur le rythme du commentaire.

\section{Cadre théorique}

Notre étude s'inscrit dans la suite des études récentes qui se sont consacrées à l'analyse des genres de discours oral, notamment avec l'objectif d'en dégager les particularités phonostylistiques, c'est-à-dire de relier des patrons prosodiques (rythmiques et intonatifs) avec des traits situationnels. Par rapport au phonogenre qui nous intéresse plus particulièrement ici, à savoir le commentaire sportif en direct, Audrit et al. (2012) ont proposé une analyse phonostylistique du genre en faisant contraster trois sports : le rugby, le football et le basketball. L'analyse phonostylistique portait aussi bien sur des mesures rythmiques (débit de parole et d'élocution, durée des syllabes, densité des syllabes accentuées...), que sur des mesures au niveau tonal (moyenne de F0, F0 range...). L'analyse a montré des différences prosodiques entre les différents sports, ou du moins une discrimination possible entre d'une part le football et le rugby, qui présentent tous les deux des variations prosodiques ponctuelles, et le basketball d'autre part, plus homogène tout au long du match. Par ailleurs cette étude proposait de différencier différents moments du jeu, en faisant non seulement une distinction entre le «color-commentary » et le « play-by-play », mais en intégrant différentes actions applicables aux trois types de sport, à travers une annotation manuelle du commentaire. L'article fait état d'une différence significative de débit entre l'action «possession », qui désigne qui (équipe ou joueur) est en possession du ballon, qui présente un débit de parole plus lent par rapport à un autre genre de discours, supposé servir d'étalon, et qui est la parole lue. De manière générale, il semble que l'étude, si elle permet de mettre en avant les différences prosodiques entre les commentaires des trois types de sports, ne définisse pas réellement de différences significatives entre les différents types d'actions commentées. On notera que l'annotation des actions est appliquée au commentaire, c'est-à-dire au discours, mais pas aux images qui amènent ce commentaire. De ce fait, l'annotation n'est pas une annotation objective des actions qui motivent le commentaire. On pourrait émettre l'hypothèse que le manque de différences prosodiques entre les différentes actions commentées du jeu reflète un manque de précision de l'annotation, qui est par ailleurs basée sur le commentaire, ou des paramètres prosodiques trop généraux pour s'appliquer à ce niveau de 
différenciation. La comparaison du genre commentaire sportif en direct avec un autre genre de discours, la parole lue, paraît peu pertinente, la caractérisation des deux genres ne reposant pas sur les mêmes critères : la parole lue s'oppose de manière générale à toute parole spontanée, mais le commentaire sportif en direct n'est pas caractérisé uniquement par son caractère spontané. Ainsi, Krazem (2011) rappelle la nécessité de prendre en compte le trait [+visuel] et [+direct]. Augendre et al. (2014) proposent un système de contraintes hiérarchisées sur le modèle de la théorie de l'optimalité. Parmi les contraintes identifiées, en plus des contraintes de genre, les auteurs insistent sur les contraintes liées au sport, c'est-à-dire au contenu commenté, supposant, comme Audrit et al. (2012) d'ailleurs, que le commentaire va varier en fonction du type de sport commenté (match, performance individuelle, course...)

Poursuivant l'idée de ces différentes études, nous considérons que le commentaire est construit selon des contraintes de genre, de média et de contenu. Les contraintes de genre sont celles qui permettent de faire du commentaire sportif en direct un genre perçu en tant que tel et différencié d'autres types de commentaires en direct, comme le commentaire d'un défilé militaire par exemple (Mathon, 2014). Les contraintes de média et les contraintes de contenu vont impacter directement sur le type de structures discursives produites par les locuteurs, ainsi qu'au niveau prosodique, sur les phonostyles. Partant de cette hypothèse, nous allons montrer l'impact du rythme du sport sur d'une part la distribution de la parole entre les deux locuteurs principaux qui co-construisent le commentaire, le journaliste et l'expert, et d'autre part sur le rythme de parole de chacun de ces deux locuteurs en fonction du contenu.

\section{Corpus et annotations}

Nous travaillons sur un corpus de commentaires sportifs en direct télévisuels (Lortal et al., 2008), et notamment dans le cadre de cette étude sur le commentaire sportif en direct du match introductif de la Coupe du Monde de Rugby 2007, entre la France et l'Argentine. Le commentaire est en français. Diffusé sur TF1, ce match était commenté par le journaliste sportif Thierry Gilardi, assisté par un expert du rugby, ancien joueur, Thierry Lacroix, et un « homme de terrain » Fabrice Landreau, également ancien joueur de rugby. Le match commenté dure 1 heure et 46 minutes environ. Il a fait l'objet d'une transcription orthographique, effectuée à l'aide du logiciel Transcriber. La transcription orthographique et le son ont été ainsi alignés à un premier niveau sur la base de segments de parole (tours de parole) séparés par des silences supérieurs ou égaux à $200 \mathrm{~ms}$ et/ou par l'alternance de parole entre les locuteurs. Le commentaire est composé de 2050 segments de parole, tout locuteur confondu.

\subsection{Annotation des actions du jeu et du commentaire}

Afin de montrer l'impact du jeu sportif sur le commentaire, nous avons repris le corpus annoté par Augendre et al.(2014) et nous avons enrichi cette annotation avec l'étiquetaage de toutes les images du match diffusées à l'attention des téléspectateurs et en partie commentées par le journaliste et l'expert. L'annotation a été faite à l'aide du logiciel Aegissub, logiciel de sous-titrage. Celui-ci nous a permis d'aligner les annotations des actions, la transcription orthographique et l'annotation du locuteur. Le tout était exportable sous la forme d'un fichier .csv.

Une passe supplémentaire d'annotations manuelles nous a permis de noter les actions effectivement commentées, en mettant en lien chaque action avec son commentaire, quand l'action était commentée. Le tableau ci-dessous synthétise la liste des actions utilisées pour l'annotation de la vidéo, et le pourcentage de commentaire par speaker pour chaque action. Les actions sont classées des plus commentées au moins commentées. Cela nous permet de distinguer les actions proéminentes, qui doivent systématiquement être commentées, comme par exemple l'essai, commenté dans tous les cas par le journaliste, des actions beaucoup moins importantes pour la compréhension du jeu, qui ne sont pratiquement pas commentées, comme par exemple la mise en ligne avant la relance suite à une touche (commentée dans $8 \%$ des cas par le journaliste uniquement). Ce ne sont pas seulement les actions de jeu qui ont été annotées sur cette vidéo, mais également les images diffusées du stade, les temps de hors-jeu... Ces images peuvent faire parfois l'objet d'un commentaire : c'est le cas par exemple des images du stade qui sont commentées par 
le journaliste dans $11.8 \%$ des cas. Nous avons également fait une distinction entre les images diffusées en direct, des images diffusées en replay et en ralenti. La condition de replay ou de ralenti va permettre une anticipation du commentaire, puisque le commentateur a déjà assisté à l'action en direct. On notera que le replay tout comme le ralenti, quand ils sont commentés, le sont presque exclusivement par l'expert, et non par le journaliste.

\begin{tabular}{|c|c|c|c|c|c|c|c|}
\hline Actions ( $\geq 50 \%)$ & Journaliste & Expert & Terrain & Actions $(\leq 50 \%)$ & Journaliste & Expert & Terrain \\
\hline essai & 100,0 & & & porté & 46,9 & & \\
\hline marqué & 100,0 & & & mise en place & 42,9 & & \\
\hline non marqué & 100,0 & & & extraction & 40,7 & 0,6 & \\
\hline petit coup de pied & 100,0 & & & passe & 38,6 & & \\
\hline tir au but & 81,3 & 6,3 & & ralenti & 9,3 & 25,6 & \\
\hline sifflet & 73,7 & 7,9 & & lâché & 34,4 & & 14,3 \\
\hline maul & 73,3 & 6,7 & & blessé & 14,3 & & \\
\hline grand coup de pied & 74,0 & & & regroupement & 25,6 & 1,2 & \\
\hline mêlée & 68,4 & & 5,3 & plaquage & 25,6 & & \\
\hline sortie & 65,5 & 3,4 & & rebond & 18,2 & & \\
\hline récupération & 64,3 & 1,6 & & stade & 11,8 & & \\
\hline introduction & 60,0 & & & mise en ligne & 8,0 & & \\
\hline réception & 56,9 & 1,3 & & hors-jeu & & & \\
\hline $\begin{array}{c}\text { lancer introduction } \\
\text { changement }\end{array}$ & 50,0 & 6,3 & & mi-temps & & & \\
\hline replay & 33,3 & & 16,7 & touché en but & & & \\
\hline
\end{tabular}

Table 1 : Liste des actions annotées, pourcentage de commentaire réparti par locuteur pour chaque action.

\subsection{Annotation «play-by-play» vs «color commentary» vs «supportaire»}

Le repérage des actions effectivement commentées nous a permis d'attribuer automatiquement à chaque segment de parole des étiquettes «play-by-play » et « color commentary » (Hartmann, 2011) représentant respectivement les segments de parole faisant référence directement aux images diffusées par opposition au discours plus explicatif, qui n'est pas lié nécessairement aux images (stratégies, biographies des joueurs). L'annotation automatique a fait l'objet d'une vérification manuelle, qui nous a permis de distinguer un troisième type de commentaire, ni description/narration des actions du jeu, ni explication favorisant la compréhension générale du jeu.

Ce troisième type porte sur des segments de parole où le locuteur semble se comporter en supporter et encourage l'une des deux équipes, et ici plus particulièrement l'équipe de France. Nous l'avons appelé « supportaire ». Il présente différentes structures linguistiques :

- Des onomatopées et des exclamations, hou la hou la, oh la la

- Des évaluations, on est bien on est bien

- $\quad$ Des injonctions, allez, allezou encore allez du soutien vite vite 
Les structures du supportaire sont souvent dédoublées. Elles sont produites presque exclusivement par l'expert et pour une certaine partie simultanément avec une production du journaliste, dans des segments présentant un chevauchement de parole.

\subsection{Méthodologie}

\subsubsection{Alignement du signal au niveau phonématique}

Le calcul sur l'ensemble de l'enregistrement des valeurs de débit des locuteurs nécessite un alignement du signal avec une transcription phonétique au niveau du phonème. Cet alignement a été produit automatiquement à l'aide du logiciel JTrans (Cerisara, Mella \& Fohr, 2009), qui à partir de la transcription orthographique effectuée avec Transcriber, a fourni une segmentation au niveau du phonème et un alignement avec la transcription phonétique, le tout sous un format Textgrid, lisible avec le logiciel d'analyse de parole Praat.

\subsubsection{Calcul du rythme de parole et du rythme du jeu}

Nous distinguons deux types de débits : un débit de parole qui mesure le nombre de syllabes prononcées par seconde sur un laps de temps donné d'une part, et un débit d'élocution qui mesure le nombre de syllabes prononcées par seconde sur le temps de parole, hors silence et pause sur de parole. Par exemple, au début du match, le journaliste dit : « pour Hernandez, avec le pied droit » (00:00:47,49-00:00:50,89). Il prononce les 9 syllabes en 3,4 secondes. Son débit de parole est 9/3,4=2,6 syllabes/seconde. Pour calculer, sa vitesse d'élocution, nous ne prenons en compte que le temps qu'il utilise pour parler. Il fait une pause de 0,92 secondes au milieu de l'énoncé. Son temps parlé est donc 3,4-0,92=2,48 secondes et de fait son débit d'élocution $9 / 2,48=3,6$ syllabes/seconde.

Pour calculer les débits de parole et d'élocution dans le corpus, nous utilisons des fenêtres centrées autour de l'instant considéré. Nous avons considéré plusieurs tailles de fenêtres pour examiner l'influence de leur dimension sur les corrélations avec les autres grandeurs temporelles, de la plus petite de $2 \mathrm{~s}$ (1s avant, $1 \mathrm{~s}$ après) à la plus grande de $24 \mathrm{~s}$.

Pour le rythme du jeu, nous utilisons une stratégie similaire à celle décrite ci-dessus pour la mesure du débit. Contrairement aux mesures de débit, nous utilisons ici des fenêtres situées avant l'instant considéré pour ne prendre en compte que des événements directement accessibles aux participants. Une fenêtre centrée prendrait en compte des événements encore à venir. Le rythme correspond au nombre d'actions par 5 secondes dans des fenêtres allant de 2s à 16s avant l'instant courant.

\section{Répartition des périodes de commentaires par rapport au rythme du jeu}

De manière assez consensuelle, il est admis que le commentaire sportif est constitué de deux types de discours :

- $\quad$ Périodes descriptives et périodes complémentaires pour Augendre et Mathon (2012) qui reprennent la distinction de Deulofeu (2000)

- «Dramatization-Description » et «Elaboration-Commentary » pour Audrit et al. (2012)

- «Play-by-play » et « Color-commentary » pour Hartmann (2011)

Ces différentes dénominations regroupent une même distinction qui est faite entre les moments où le commentaire est directement motivé par les images diffusées, et qu'il est constitué d'une narration synchronisée des actions visualisées, et les moments où le commentaire est désynchronisé par rapport à ce qui se passe sur le terrain, et est constitué de phases plus explicatives, pourtant sur le jeu en général, les règles, les stratégies... 
Nous reprenons pour notre part la dénomination de Hartmann (2011) et nous avons distingué les périodes de «play-by-play» des périodes de «color-commentary», en se basant sur l'annotation des actions commentées (cf. section 3.2.). Cette annotation nous permet d'examiner quels sont les moments où chacun de ces types de commentaires apparaît, et ce en relation avec la nature des actions et le rythme du jeu. Un graphe nous permet de représenter sur l'ensemble du match, le rythme du jeu (en bleu), les périodes de «play-by-play »(en vert) et les périodes de « color-commentary» (en rouge).

Le graphe 1 ci-dessous représente un moment du jeu où l'on voit le rythme de jeu accélérer progressivement, à partir de 980 (La ligne horizontale représente la ligne de temps en secondes.). Avec un léger décalage, on voit comment la ligne rouge représentant les périodes de «color-commentary » est remplacée par la ligne verte des périodes de «play-by-play ». Le rythme du jeu s'accélère ici quand la mêlée est suivie de plusieurs cycles d'actions rapides regroupées sous l'événement «pick-and-go »: extraction, passe, réception, porté, plaquage, regroupement, extraction... L'événement « pick-and-go » dans son ensemble fait l'objet d'un commentaire (toutes les actions individuelles n'étant pas forcément commentées). Cette période se termine par une mise en touche. Celle-ci marque la fin du rythme rapide. Durant la préparation nécessaire à la remise en touche, préparation qui ne présente pas beaucoup d'intérêt du point de vue de la narration, le «color-commentary » va prendre la place du «play-by-play », avec toujours un léger décalage par rapport au rythme du jeu. Le journaliste termine son « play-by-play » avant que l'expert embraye sur le « color-commentary ».

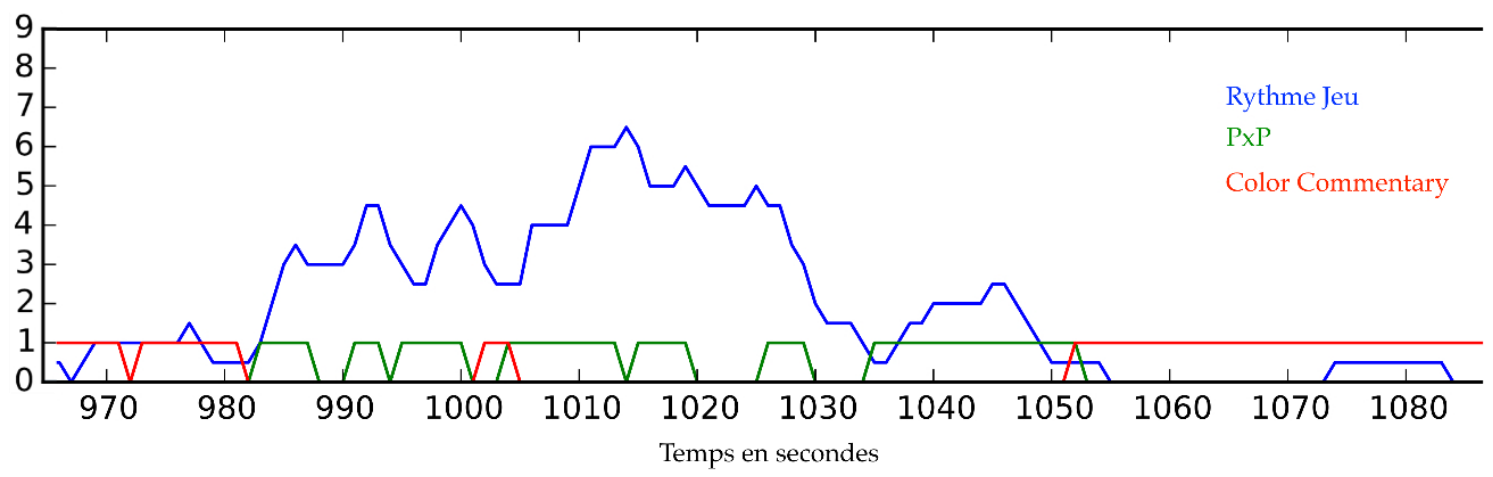

Graphe 1: Rythme de jeu (en bleu) et type de commentaires, «play-by-play» (en vert) et «colorcommentary » (en rouge)

Le graphe 2 montre un autre moment d'accélération du rythme du jeu, où ici la période de jeu rapide, encore un «pick-and-go », voit une répartition équilibrée des deux types de commentaires «play-byplay » et «color-commentary ». L'enchaînement des actions dans le 《pick-and-go » présentant un caractère répétitif, le « play-by-play » ne s'attache pas à toutes les actions en détails et laisse la place pour du « color-commentary » inséré à intervalles réguliers. Le commentaire durant cette période rapide reste tout de même majoritairement du « play-by-play ».

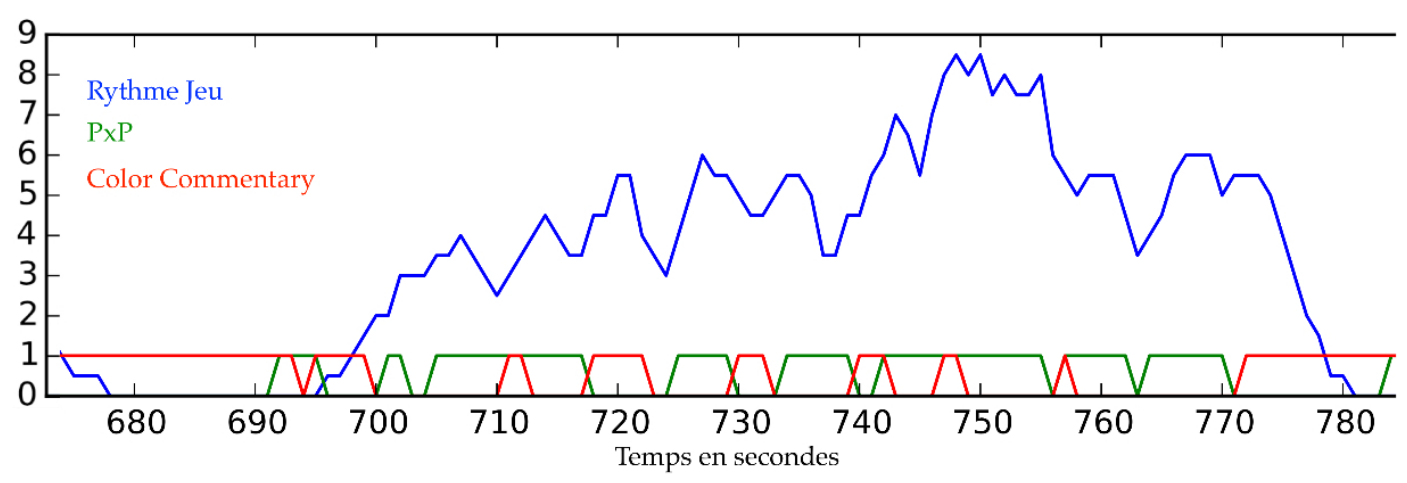


Graphe 2 : Rythme de jeu (en bleu) et alternance de «play-by-play » (en vert) et de « color-commentary » (en rouge)

\section{$5 \quad$ Répartition de la parole entre les trois locuteurs}

Cette section s'intéresse à montrer comment le rythme et le contenu du jeu détermine la prise de parole du journaliste et de l'expert en fonction de leurs compétences linguistiques respectives. Nous avons montré dans la section précédente comment le rythme du jeu influait sur le type de commentaires produit, «playby-play » ou « color-commentary ». De manière générale, on observe qu'un rythme de jeu lent favorise le « color-commentary » tandis qu'un rythme de jeu rapide favorise lui le « play-by-play ».

Si l'on regarde maintenant les prises de parole en fonction du rythme de jeu, on s'aperçoit que le rythme de jeu lent est favorable à une prise de parole de l'expert. Le graphe 3 ci-dessous montre l'évolution du rythme du jeu (en bleu) et celle du débit de parole de l'expert (en vert). Un peu avant 670, une pénalité a été sifflée, marquant la fin d'une période de jeu rapide de «pick-and-go ». Pendant la phase de mise en place de la mêlée, le rythme de jeu est fortement ralenti. Cette période coïncide avec la prise de parole de l'expert. Dès que le rythme de jeu accélère à nouveau (vers 690), l'expert termine son intervention.

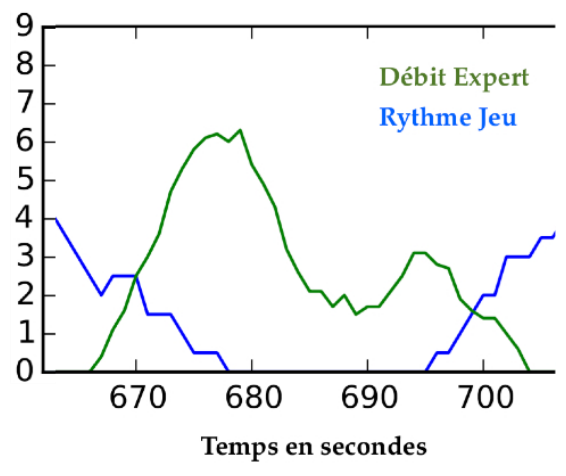

Graphe 3 : Rythme de jeu (en bleu) et rythme de parole de l'expert (en vert)

Les périodes de rythme de jeu lent semblent donc favoriser la prise de parole par l'expert pour un type de commentaires qui relève plus du «color-commentary». Nous avons en effet calculé le nombre d'interventions de chaque locuteur pour les périodes de «play-by-play » et de « color-commentary ».

\begin{tabular}{|c|c|c|}
\hline & «Play-by-play $»$ & «Color-commentary » \\
\hline Journaliste & 1342 & 1767 \\
\hline Expert & 603 & 1479 \\
\hline
\end{tabular}

Table 2 : Nombre de segments de parole répartis par locuteur et par type de commentaire.

Le tableau 2 montre que l'expert intervient beaucoup plus dans le «color-commentary » que dans le « play-by-play» (plus du double d'interventions), même si le journaliste est celui qui fait le plus d'interventions tout type de commentaires confondu.

Le graphe 4 montre l'alternance des prises de parole du journaliste (ligne rouge) et de l'expert (ligne verte) dans une période de «color-commentary » (ligne bleu turquoise). Le rythme de jeu (ligne bleu foncé) est lent, ce qui favorise l'apparition de «color-commentary », qui est réparti de manière assez régulière entre les deux locuteurs. 


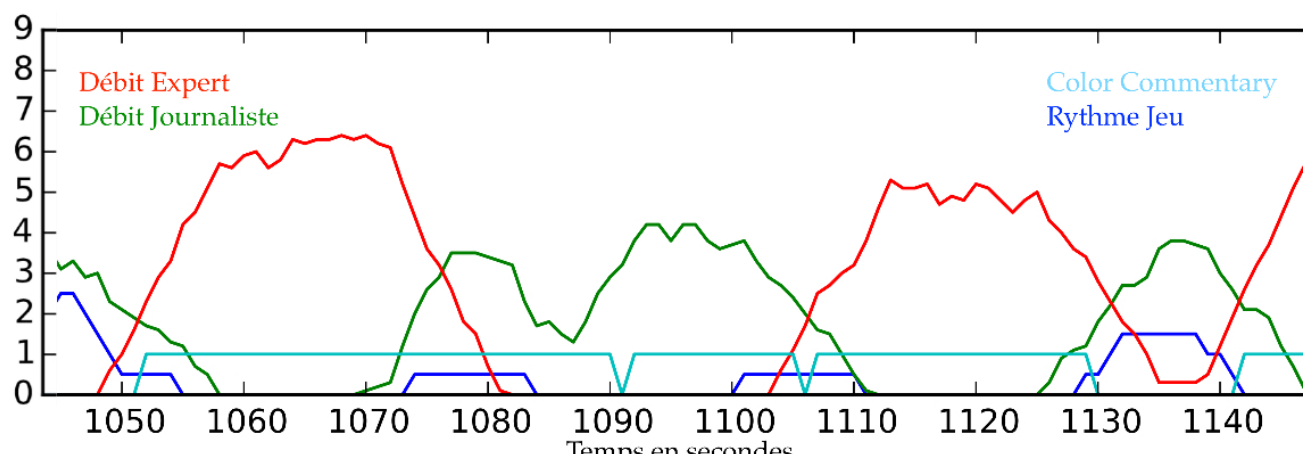

Graphe 4 : Répartition de la parole entre le journaliste (en rouge) et l'expert (en vert) pour une période de « color-commentary » (en bleu turquoise)

Si le rythme de jeu lent favorise le «color-commentary », le rythme de jeu rapide est le lieu privilégié du «play-by-play ». Dans ces phases rapides où le type émergent est le «play-by-play », c'est le journaliste qui produit l'essentiel des interventions. Le graphe 5 ci-dessous montre ainsi l'accélération du rythme de jeu (ligne bleu) coïncidant avec un événement de pick-and-go, et la prise de parole presque parallèle du journaliste (ligne verte). Sans surprise, le type de commentaires produit sur cette même période est du «play-by-play » (ligne rouge) : l'expert ne prend pas part au commentaire ici, le rythme rapide et le «play-by-play » semblant correspondre presque exclusivement au journaliste.

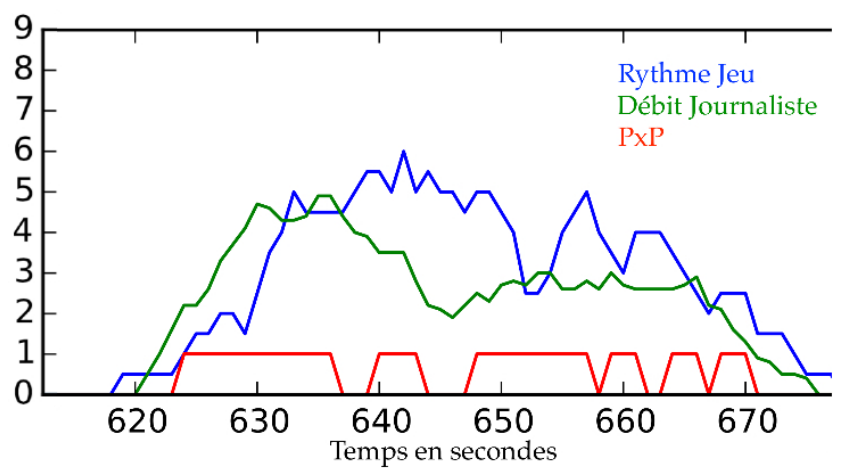

Graphe 5: Rythme de jeu (en bleu) et débit de parole du journaliste (en vert) pendant une période de «play-by-play » (en rouge)

\section{Rythme de jeu et rythme de parole}

Nous présentons dans cette section les principales observations faites sur la base des mesures de débit (débit de parole et débit d'élocution) qui ont été effectuées sur l'ensemble de l'enregistrement, en distinguant bien sûr les interventions des différents locuteurs. Nous relions les variations du rythme du jeu avec les variations de débit de parole et les types de commentaires «play-by-play» et «colorcommentary ».

Considérant que le journaliste intervient de manière privilégiée dans les périodes où le rythme de jeu est rapide pour produire du «play-by-play », nous pouvons émettre l'hypothèse que son rythme de parole est globalement plus rapide que celui de l'expert, ce qui lui permet de s'adapter aux périodes de jeu rapides. Les mesures de débit montrent en réalité que l'expert parle en moyenne plus vite que le journaliste. Si on distingue les mesures de débit en «play-by-play » et en « color-commentary » (cf. Table 3), on s'aperçoit 
que l'expert parle effectivement avec un débit plus important que le journaliste dans le «colorcommentary », 1.95 contre 1.53 pour le débit de parole et 2.21 contre 1.82 pour le débit d'élocution.

Dans le «play-by-play », la tendance est inversée. C'est le journaliste qui parle le plus vite, que ce soit en termes de débit de parole ( 2.18 contre 0.9 pour l'expert) ou de débit d'élocution (2.52 contre 1.02$)$. Le débit du journaliste dans le « play-by-play » est plus de deux fois plus rapide que celui de l'expert.

\begin{tabular}{|l|c|c|c|c|}
\hline \multirow{2}{*}{} & \multicolumn{2}{|c|}{ «Play-by-play } & \multicolumn{2}{c|}{ «Color-commentary » } \\
\cline { 2 - 5 } & Journaliste & Expert & Journaliste & Expert \\
\hline Débit de parole & 2.18 & 0.9 & 1.53 & 1.95 \\
\hline Débit d'élocution & 2.52 & 1.02 & 1.82 & 2.21 \\
\hline
\end{tabular}

Table 3 : Débit de parole et débit d'élocution moyens par locuteur et par type de commentaires

Il est intéressant de noter par ailleurs que si le rythme de parole du journaliste est plus important dans le «play-by-play » que dans le « color-commentary », le rythme de parole de l'expert présente la tendance inverse, c'est-à-dire une augmentation du «play-by-play» au «color-commentary ». La première observation semble cohérente avec les observations qui ont été faites dans les sections précédentes, qui montraient que le «play-by-play » semblait plus présent dans les périodes de rythme rapide et que c'était le journaliste qui intervenait de manière privilégiée dans ces phases-là. En revanche, le «colorcommentary » ne se présente que dans les phases lentes de jeu. Il semblerait cohérent que l'expert ait de fait le temps de produire son commentaire et qu'il n'ait pas la nécessité d'augmenter son débit de parole. En examinant la durée moyenne des tours produits par le journaliste et par l'expert, on note que l'expert fait en moyenne des tours plus longs (2.21s pour un écart-type de 1.62) que le journaliste (1.88s pour un écart-type de 1.03). Les graphes 6 et 7 ci-dessous montrent la répartition des tours de paroles respectivement du journaliste et de l'expert en fonction de la durée des tours. Ces graphes montrent que proportionnellement au nombre total de tours produits par chacun des locuteurs, indépendamment du type de commentaires, l'expert a une plus grande quantité de tours dont la durée est supérieure à 4 secondes. De manière générale, l'expert produit des tours plus longs que le journaliste. L'expert a plus de choses à dire que le journaliste et il le dit avec des structures plus longues et plus complexes. Ainsi, pour réussir à adapter ces structures à la fenêtre de temps dont il dispose, il est obligé d'accélérer son débit de parole.

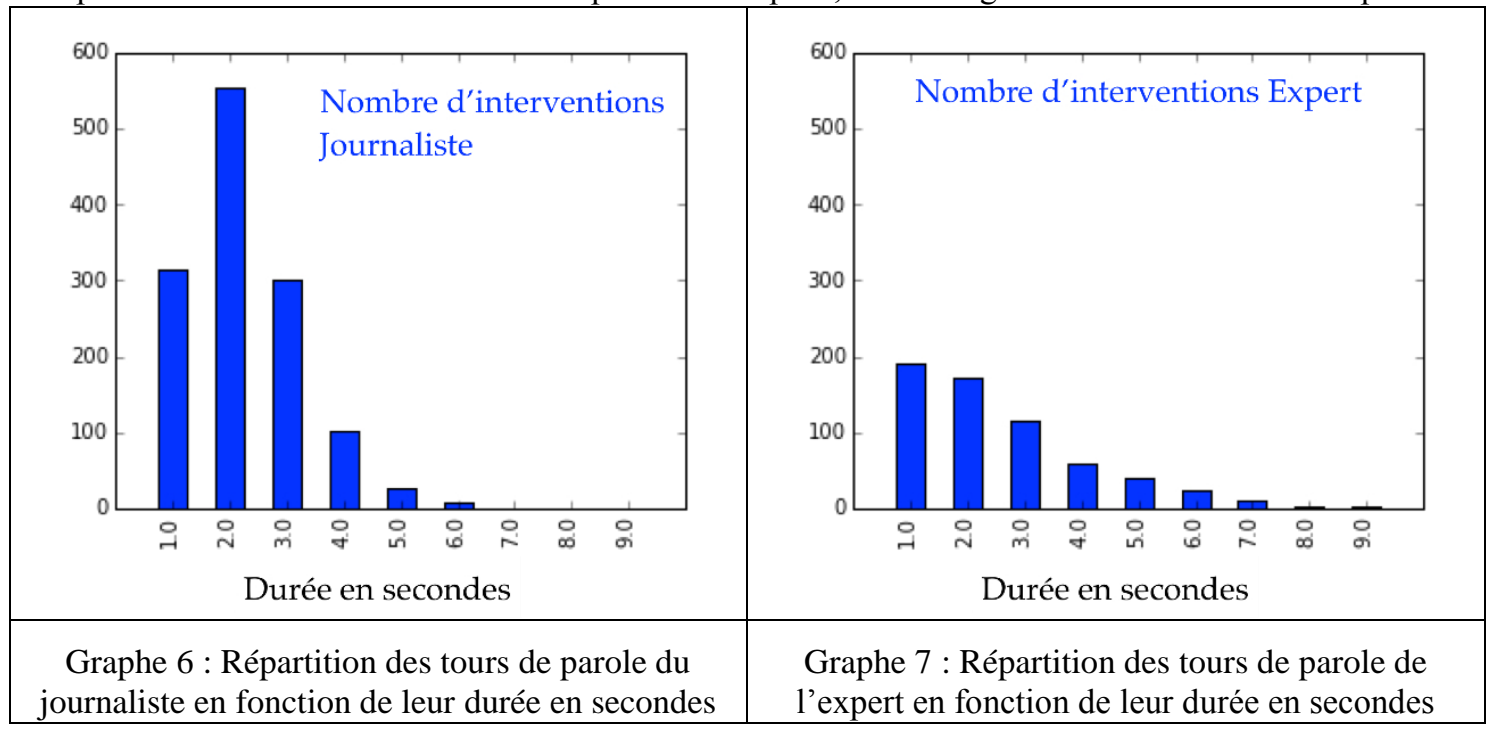

Notre seconde hypothèse concerne la corrélation entre le rythme du jeu et le rythme de parole du journaliste : dans la mesure où le journaliste est celui qui assure la majorité des commentaires de type 
«play-by-play », on peut émettre l'hypothèse que son rythme de parole va s'adapter au rythme du jeu qu'il décrit. Ainsi, quand le rythme de jeu augmente, on peut supposer que le rythme de parole du journaliste augmente également, avec un léger temps de décalage. L'observation des données montre que cette hypothèse n'est pas tout le temps vérifiée. Ainsi, certaines périodes de jeu rapides sont commentées dans un débit plus lent. Le graphe 8 montre une progression inverse du rythme de parole du journaliste (ligne verte) et du rythme du jeu (ligne bleue). A 1370, alors que le rythme du jeu commence à augmenter, le rythme de parole du journaliste entame une progression inverse et semble décliner, pour atteindre son niveau le plus bas à 1390. Ce point dans le temps correspond aussi au pic du rythme de jeu. On observe une corrélation négative du rythme du jeu et du rythme de parole du journaliste.

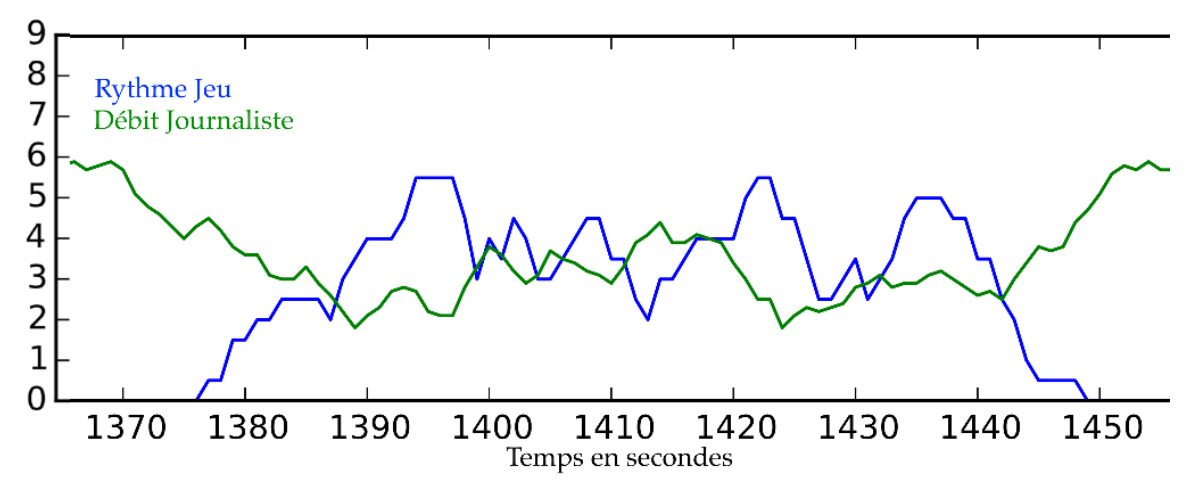

Graphe 8 : Rythme de jeu (en bleu) et débit de parole du journaliste (en vert) : exemple de progression inverse

Si le journaliste n'adapte pas son rythme de parole au rythme du jeu dans le « play-by-play », il doit sans doute avoir une autre stratégie d'ajustement de son discours à l'action. L'examen des structures linguistiques peut nous permettre de comprendre cette stratégie. Ainsi, pour la période qui court de 1390 à 1430, les structures employées par le journaliste, qui est en train de narrer, sont relativement courtes, comme par exemple :

1390,27 -Ledesma

1393,64 - Hernandez

1394,76 - et encore une chandelle pour tester

1397,48 - Heymans

1399,48 - ouais

1400,08 - qui cette fois

1401,20 - la chandelle était trop longue et qui cette fois peut effectuer un arrêt de volée

1406,83 - par contre sa relance trouve euh Corleto

1410,83 - encore en l'air pour Corleto

1412,60 - histoire de remettre en jeu tous ses partenaires

1414,72 - il a pris le ballon l'ami Corleto

1416,72 - il peut jouer avec Contepomi

1420,16 - et le soutien qui arrive des Argentins

1422,48 - en rafale

1425,48 - ballon libéré

1428,24 - voilà pour euh

1429,68 - Pichot

1430,79 - Felipe Contepomi au pied

Entre 1390 et 1400, où le rythme de parole est bas, le journaliste emploie des structures très courtes, constituées notamment de noms propres qui désignent les joueurs. On distingue ensuite une deuxième période, de 1400 à 1420, durant laquelle le rythme de parole augmente : les structures ici sont plus longues, constituées de propositions verbales indépendantes ou subordonnées. A partir de 1420, le rythme 
de parole diminue à nouveau et parallèlement, les structures employées sont beaucoup plus courtes, groupe nominal ballon libéré, des noms propres Pichotou encore un groupe nominal prépositionnel en rafale. A l'examen des structures linguistiques produites par le journaliste dans les périodes de «play-byplay » où on trouve une corrélation négative entre le rythme du jeu et le rythme de parole, il semblerait que l'ajustement du discours du journaliste au jeu se fasse non pas à travers l'augmentation du débit de parole, mais en choisissant des structures linguistiques plus courtes, spécifiques par ailleurs du commentaire sportif (Deulofeu, 2000 ; Krazem, 2011 ; Augendre et al., 2016).

De manière à examiner le lien entre le rythme du jeu et le rythme de parole des commentaires des deux locuteurs principaux sur l'ensemble du match, nous avons calculé la corrélation entre les deux rythmes en testant des intervalles de différentes durées sur le rythme du jeu et le rythme de parole ( 8 intervalles de 2 à 16 secondes). Nous avons distingué les locuteurs, le journaliste et l'expert, et le type de commentaires, «play-by-play » et « color-commentary ». Nous avons été amenés également à distinguer la première mitemps et la seconde mi-temps, les corrélations semblant différer de l'une à l'autre.

Sur la première mi-temps et en ce qui concerne le «play-by-play » les deux locuteurs présentent un comportement différent du point de vue du débit. En effet, alors que le journaliste présente une corrélation positive avec un coefficient de $58 \%$ dans la fenêtre de rythme de jeu de $8 \mathrm{~s}$, l'expert présente lui une corrélation négative avec un coefficient de $-57 \%$ dans les fenêtres de rythme de jeu de 10 s et 12 s. On remarque donc d'une part que les locuteurs ont un comportement opposé du point de vue de l'ajustement du débit au rythme du jeu, puisque le journaliste ajuste son débit au rythme du jeu dans le «play-by-play », tandis que l'expert semble ajuster son débit de parole à l'opposé du rythme du jeu (corrélation négative). Mais d'autre part, les intervalles de rythme de jeu auxquels correspondent les optimaux de corrélation ne sont pas les mêmes d'un locuteur à l'autre. Toujours en première mi-temps, mais dans le «color-commentary » cette fois, les optimaux de corrélation apparaissent cette fois dans le même intervalle de rythme de jeu pour les deux locuteurs (Rythme de Jeu,12s). Pour le journaliste, le coefficient de corrélation est moins important que dans le «play-by-play » mais reste positif à $41 \%$. Pour l'expert, le coefficient de corrélation est négatif à $-38 \%$.

En seconde mi-temps, le rythme de parole en «play-by-play » est moins corrélé au rythme de jeu :

- Le journaliste présente une corrélation positive avec un coefficient de seulement $33 \%$ en fenêtre de rythme de jeu de $8 \mathrm{~s}$;

- L'expert présente une corrélation toujours négative, mais avec un coefficient de corrélation à seulement $-29 \%$ en fenêtre de rythme de jeu de 6 s.

Le color-commentary ne présente pas de régularité remarquable.

Pour essayer de comprendre ce qui distinguait la première mi-temps de la seconde, et expliquer ces différences de corrélation entre le rythme du jeu et le rythme de parole, nous avons examiné les comportements discursifs des deux locuteurs et les annotations de la vidéo, en comparant les deux mitemps. Les deux mi-temps ne présentent pas tout d'abord le même nombre d'actions : la première mitemps présente 850 actions contre 932 en deuxième mi-temps. Pour autant, et contrairement à ce qu'on aurait pu croire, c'est dans la première mi-temps qu'il y a le plus d'actions commentées («play-byplay »), 1099 contre 907 en deuxième mi-temps. Parallèlement, le nombre de «color-commentary » augmente significativement au cours du match, puisqu'on passe de 1395 «color-commentary » en première mi-temps à 1967 en seconde mi-temps. Le jeu de la première mi-temps, alors qu'il est moins varié, est pourtant plus commenté. On peut expliquer cette apparente contradiction par le déroulement du jeu en lui-même durant ce match et les enjeux liés à ce match au sein de la compétition. Le match oppose l'Argentine à la France dans le premier match de poule de cette Coupe du Monde de Rugby. Assez rapidement, les Argentins dominent le jeu et dès le début de la seconde mi-temps, il semble de plus en en plus évident que les Français ne vont pas réussir à combler l'écart de points pour gagner le match. Les commentateurs vont donc adapter leur commentaire et plutôt que de mettre en avant les actions du match, qui ne sont pas nécessairement à l'avantage des Français, ils vont plutôt présenter les enjeux pour chaque équipe dans la compétition, les stratégies pour gagner des points afin d'être qualifiés en quart de finale... De ce fait, une plus grande partie des productions est constituée de «color-commentary ». C'est aussi 
dans cette seconde mi-temps que l'on trouve la majorité des occurrences de «supportaire » : l'équipe de France étant en difficulté, les commentateurs et notamment l'expert produisent des encouragements à l'attention de l'équipe.

\section{Conclusion}

Notre travail repose sur l'idée que le commentaire sportif en direct est contraint quant au contenu, au sens où une partie du commentaire a un rapport direct avec les actions commentées, que le média de diffusion soit d'ailleurs la télévision (comme c'était le cas ici) ou la radio. Nous supposons donc que le contenu commenté, et ici son rythme, influe sur la manière dont il est commenté, et ici plus spécifiquement sur le rythme du commentaire. Nous avons donc entrepris cette étude avec l'idée, peut-être un peu naïve, que le rythme du jeu influençait directement le rythme du commentaire.

Nous avons montré qu'en réalité il n'y a pas de corrélation simple entre le rythme du jeu et le rythme de parole, et ce aussi bien pour le journaliste que pour l'expert. On trouve bien sûr ponctuellement des lieux de correspondance, c'est-à-dire des endroits du discours où le rythme de parole, le plus souvent du journaliste, semble correspondre parfaitement avec le rythme du jeu. On trouve également et de manière symétrique des moments du discours où le rythme de parole, celui de l'expert en l'occurrence, se comporte à l'inverse du rythme du jeu. Mais, si l'on regarde la correspondance sur l'ensemble du match entre rythme de parole et rythme du jeu, on s'aperçoit que la corrélation n'est pas systématique, y compris pour le journaliste. Ainsi, sans doute, la compétence du journaliste à adapter son discours au jeu ne tient pas uniquement dans les variations de débit qu'il est capable de faire. Les études précédentes sur les structures syntaxiques typiques du «play-by-play» nous amènent à penser que la compétence du journaliste est mixte : il peut jouer à la fois sur son rythme de parole mais également sur les structures linguistiques qu'il choisit d'employer pour adapter son commentaire au jeu. A l'inverse, il semblerait que le débit de parole soit une variable d'ajustement pour permettre aux deux locuteurs, aussi bien le journaliste que l'expert, de placer un maximum de « color-commentary », avec des structures syntaxiques plus longues et plus complexes.

Enfin, l'importance de la corrélation entre le rythme de jeu et le rythme de parole semble sujette à des variations tout au long du match et notamment entre la première et la seconde mi-temps. Le développement macro du jeu, le fait qu'une équipe semble dominer l'autre, la dynamique générale du jeu... tous ces aspects vont influer sur les stratégies discursives des commentateurs, qui vont choisir de produire plutôt du «play-by-play » ou plutôt du « color-commentary ». Ces choix stratégiques au niveau de l'organisation du discours influent à leur tour sur la correspondance entre rythme de jeu et rythme de parole. Un travail d'analyse conjointe du développement du match et du commentaire en parallèle pourrait ici sans doute confirmer nos hypothèses.

\section{Références bibliographiques}

Audrit, S., Prsir, T., Auchlin, A. \& Goldman, J.-P.(2012). Sport in the Media: A Contrasted Study Of Three Sport Live Media Reports With Semiautomatic Tools. In Qiuwu, M., Hongwei, D. \& Hirst, D. (Eds.), Proceedings Of The 6th International Conference On Speech Prosody, Vol. 1, Shanghai : Tongji University Press, 127130.[http://www.speechprosody2012.org/page.asp?id=157]

Augendre, S., Mathon, C. (2012). Comment l'information à transmettre influence-t-elle les constructions référentielle et syntaxique du commentaire sportif en direct ? In Actes du CMLF 2012 - 3ème Congrès Mondial de Linguistique Française, pp. 2027-2040, publié par EDP Sciences (www.linguistiquefrancaise.org). http://dx.doi.org/10.1051/shsconf/20120100274

Augendre, S., Mathon, C., Boye, G., Kupsc, A. (2014). Influence des contraintes extra-linguistiques sur le discours : cas du commentaire sportif télévisé. In Actes du CMLF 2014 - 4ème Congrès Mondial de Linguistique Française, pp.1905-1924, publié par EDP Sciences (www.linguistiquefrancaise.org). http://dx.doi.org/10.1051/ shsconf/20140801381 
Augendre, S., Kupsc, A., Mathon, C., Boyé, G.(2016, soumis). Live TV Sports Commentaries: Specific Syntactic Structures and General Constraints. In Proceedings of Journée ConSciLa "Grammaire des genres et des styles : quelles approaches privilégier ?’, Paris, 16 janvier 2015.

Cerisara, C., Mella, O. and Fohr, D. (2009). JTrans, an open-source software for semi-automatic text-to-speech alignment.In Proceedings of INTERSPEECH2009, Brighton.

Deulofeu, J. (2000). Les commentaires sportifs constituent-ils un "genre", au sens linguistique du terme ? In Bilger, M. (ed.), Corpus: Méthodologie et applications linguistiques. Paris : Champion.

Hartmann, C. (2011). Pre-fabricated Speech Formulas as Long-term Memory Solutions to Working Memory Overload. Thèse de Doctorat, Université de Zurich.

Krazem, M. (2011). Représenter les relations entre grammaire et genres de discours: exemple des commentaires sportifs. Les genres de discours vus par la grammaire, LINX 64-65, pp. 45-68.

Lortal, G., Mathon, C.(2008). Motion and Emotion or How to Align Emotional Cues with Game Actions. In Proceedings of EMOT Worshop, LREC 2008, Marrakech.

Mathon, C. (2014). Perception des phonostyles et représentativité du phonogenre : le cas du commentaire sportif en direct. Nouveaux cahiers de linguistique française, 31, pp. 93-103. Actes des Journées SWIP 3, Genève, Septembre 2014. 